\title{
Drug resistance analysis of gefitinib-targeted therapy in non-small cell lung cancer
}

\author{
SHULIANG LIU ${ }^{1 *}$, HONGJI YANG ${ }^{2 *}$, XINGPING GE ${ }^{3}$, LINGFEI SU ${ }^{3}$, \\ AIFENG ZHANG ${ }^{1}$ and LIANG LIANG ${ }^{4}$ \\ ${ }^{1}$ Department of Thoracic Surgery, Yantaishan Hospital, Yantai, Shandong 264001; ${ }^{2}$ Department of Respiratory Medicine, \\ Affiliated Hospital of Taishan Medical University, Tai'an, Shandong 271000; ${ }^{3}$ Department of Radiotherapy, \\ Yantaishan Hospital, Yantai, Shandong 264001; ${ }^{4}$ Oncology Center, Sichuan Provincial People's Hospital, \\ Chengdu, Sichuan 610000, P.R. China
}

Received April 21, 2016; Accepted September 20, 2016

DOI: $10.3892 / \mathrm{ol} .2016 .5171$

\begin{abstract}
The aim of the study was to examine the drug resistance analysis of gefitinib-targeted therapy in non-small cell lung cancer (NSCLC). In total, 156 NSCLC patients without surgical treatment were selected, including 117 cases of adenocarcinoma (75\%), to receive single gefitinib $0.25 \mathrm{~g}$ /day or combined with platinum chemotherapy. Computed tomography was used to evaluate tumor growth for the response and non-response groups. The chemotherapy regimen was changed or combined with radiotherapy in the non-response group. Tumor progression or metastasis in the response group was considered as the generation of drug resistance. The chemotherapy regimen was altered in the response group. Eleven cases had tumor response in the non-response group after the chemotherapy regimen was adjusted (20\%), 33 cases had complete response (CR) (32.7\%), 44 cases had partial response (PR) (43.6\%), and 24 cases had stable disease (SD) (23.8\%) in the response group. The drug resistance rates of $\mathrm{CR}, \mathrm{PR}$, and $\mathrm{SD}$ showed no significant difference $(\mathrm{P}>0.05)$. However, the drug-resistant time of $\mathrm{CR}$ was significantly delayed and the difference was statistically significant $(\mathrm{P}<0.05)$. The response rates of $\mathrm{CR}, \mathrm{PR}$, and $\mathrm{SD}$ patients regaining the response rate showed no statistical significance after the chemotherapy regimen was adjusted, and the difference was not statistically significant $(\mathrm{P}>0.05)$. In conclusion, gefitinib-targeted therapy in NSCLC showed certain drug resistance, which may not be related to the response.
\end{abstract}

Correspondence to: Dr Liang Liang, Oncology Center, Sichuan Provincial People's Hospital, 32 West Second Section First Ring Road, Chengdu, Sichuan 610000, P.R. China

E-mail: liang_liang_1212@163.com

${ }^{*}$ Contributed equally

Key words: gefitinib, non-small cell lung cancer, drug resistance, response

\section{Introduction}

Gefitinib is a targeting drug of epidermal growth factor receptor-tyrosine kinase inhibitors (EGFR-TKIs). It is mainly used in the treatment of progressive-stage non-small cell lung cancer (NSCLC) patients who received platinum as basic chemotherapyt, and it was sensitive to adenocarcinoma in non-smoking Asian women. Gefitinib may be considered a first-line therapy regimen (1).

The overall effective rate of EGFR gene mutation was $40-65 \%$ and the overall effective rate of the non-mutation gene was $5-13 \%$ (2). Additionally, $\sim 50-75 \%$ of patients who were sensitive to gefitinib earlier appeared to acquire drug resistance in 5-10 months, indicating tumor progression or metastasis (3). At present, it is generally considered that drug resistance is related to secondary EGFR gene mutation, drug transportation, EGFR/Met gene amplification and the signal pathway (4). Many cell experiments in vitro verified this from different aspects. However, to the best of our knowledge, there are no animal and clinical studies in vivo $(5,6)$. Thus, it was not clear whether tumor progression was the generation of drug resistance or natural tumor process.

The clinical effect and drug resistance analysis of gefitinib targeted therapy in the treatment of NSCLC in Yantaishan Hospital (Shandong, China) is summarized in the study, to determine the underlying drug resistance mechanism.

\section{Patients and methods}

Patient data. In total, 156 cases of NSCLC patients in Yantaishan Hospital without surgical indication or rejecting operation were continuously selected from January, 2011 to June, 2015. The underlying pulmonary diseases, such as chronic obstructive pulmonary disease, respiratory failure, heart, liver, kidney or other organ insufficiency, intolerance to gefitinib or other chemotherapeutic drugs, continuous chemotherapy for $<1$ month, poor compliance, and incomplete follow-up data, were excluded. All patients were followed up for 6 months.

The study was approved by the Ethics Committee of Yantaishan Hospital, patients or their families. The patients 
were treated with single gefitinib (AstraZeneca, Cambridge, UK) $0.25 \mathrm{~g} /$ day or combined with platinum chemotherapy. Computed tomography was used to evaluate the tumor growth in 1 month. According to the effectiveness evaluation criterion, the maximum tumor diameter was increased in the non-response group, in a total of 55 cases (35.3\%). There were 101 cases in the response group. There were 24 cases of males and 31 cases of females in the response group, aged 46-77 years, average $56.9 \pm 13.7$ years, including 13 cases of squamous carcinoma, 42 cases of adenocarcinoma, 8 cases of stage II, 32 cases of stage III, and 15 cases of stage IV. There were 38 cases of males and 63 cases of females in the response group, aged 44-76 years, average $54.3 \pm 12.8$ years, including 40 cases of squamous carcinoma, 71 cases of adenocarcinoma, 16 cases of stage II, 68 cases of stage III, and 17 cases of stage IV. The gender, age, tumor classification, and stage showed no statistical significance between the two groups $(\mathrm{P}>0.05)$.

Research methods. After the chemotherapy regimen was altered or combined with radiotherapy in the response group, the tumor progression or metastasis was considered to be drug resistance in the response group. The patients were continuously observed. The response rate was analyzed in the non-response group after the chemotherapy regimen was adjusted. The drug-resistant time and drug resistance rate were in the response group. The effectiveness evaluation criteria were tumor diameter shrinking by $>50 \%$ was considered as complete response (CR), shrinking by $>10 \%$ was considered as partial response (PR), shrinking by $<10 \%$ was considered as stable disease (SD) and not shrinking was considered as progression (PD).

Statistical analysis. The data were analyzed using SPSS 19.0 statistical software (IBM, Armonk, NY, USA). The quantitative data were expressed using mean \pm standard deviation. The Student's t-test was used to compare the difference among the groups. The qualitative data were expressed using the case number or percentage. The $\chi^{2}$ test was used to compare the difference among the groups; $\mathrm{P}<0.05$ was considered to indicate a statistically significant difference.

\section{Results}

Response rates in the two groups. Eleven cases had tumor response in the non-response group after the chemotherapy regimen was altered for 1 month (20\%), including 4 cases of PR and 7 cases of SD. Thirty-three cases had CR (32.7\%), 44 cases had PR (43.6\%), and 24 cases had SD in the response group (23.8\%).

Drug resistance in the reaction group. The drug-resistant time was 2-6 months in the response group. The median time was 4.0 months. Ten cases had drug resistance in CR patients $(30.3 \%, 10 / 33)$. The resistant time was 4-6 months and the median time was 5 months. Fifteen cases had drug resistance in PR patients $(34.1 \%, 15 / 44)$. The drug-resistant time was 2.5-5.0 months and the median time was 3.5 months. Seven cases had drug resistance in SD patients $(29.2 \%, 7 / 24)$. The drug-resistant time was 2-4.5 months and the median time was 2.8 months. The drug resistance rates of CR, PR and
SD showed no significant difference $(\mathrm{P}>0.05)$. However, the drug-resistant time was significantly delayed and the difference was statistically significant $(\mathrm{P}<0.05)$.

Response rate in the response group after the chemotherapy regimen was adjusted. After the chemotherapy regimen was adjusted, 2 cases regained the response in CR patients $(20 \%$, 2/10), 2 cases regained response in PR group (13.3\%, 2/15), and 1 case regained response in SD patient $(14.3 \%, \mathrm{SD})$. There was no significant difference between the groups $(\mathrm{P}>0.05)$.

\section{Discussion}

Secondary mutation theory considered that EGFR gene exon 20 occurred to the secondary mutation in the gefitinib treatment process, resulting in threonine in EGFR790 locus being substituted by methionine (7). Threonine was located outside the tyrosine kinase contacting core reaction region and the hydrogen bond with high affinity formed with the adjacent gefitinib anilino, to ensure the antitumor effect. Once T790M mutation occurred, one larger amino acid side chain was introduced in this locus, to constitute the steric hindrance, influencing the formation of hydrogen bond between tyrosine kinase and gefitinib, eventually leading to gefitinib not combining with tyrosine kinase. Takeda et al (8) and Inukai et al (9) found that T790M mutation was detected in only $0.5 \%$ of patients receiving gefitinib. T790M highly sensitive mutant polymerase chain reaction detection showed that among 7 patients not reacting to gefitinib treatment, 3 patients had T790M mutation, and T790M mutation was not detected in 19 patients reacting to gefitinib treatment. Onitsuka et al (10) found that among 10 patients receiving secondary gefitinib resistance, T790M mutation was detected in 7 patients.

Some patients had gefitinib resistance by activating the downstream signaling pathway and bypassing EGFR, which is called the 'bypass activation pathways'. Engelman et al (11) found that among 18 cases of drug-resistant lung cancer cell lines induced by gefitinib, the gene amplification was detected in 4 cases. HER3 (ErbB3) phosphorylation was induced to persistently activate the PI3K/Akt pathway, resulting in secondary gefitinib resistance. It was confirmed in a variety of tumors that the maladjusted Met signaling contributed to tumorigenesis by activating mutation (such as papillary nephroblastoma) (12) or by high-level amplification (such as gastric cancer) (13).

In addition, the change of the tumor microenvironment was an important cause of drug resistance. Receptor tyrosine kinase and integrin $\beta-1$ was able to activate Akt through different pathways. The PI3K/Akt pathway played an important role in the anti-apoptotic signal transduction to cause drug resistance by regulating the apoptosis of tumor cells (14). However, 60-70\% of secondary EGFR-TKIs resistance was associated with the secondary mutation theory and Met gene amplification. Other mechanisms of drug resistance may be related to the different influence of factors including smoking, gender, race and pathological type.

The covalent binding of irreversible EGFR inhibitor with EGFR was able to overcome the binding barriers of EGFR 
with T790M mutation. In in vitro experiments, the cell line acquiring drug resistance maintained sensitivity to the irreversible EGFR inhibitor using first-generation EGFR-TKIs $(15,16)$. At present, partial irreversible EGFR inhibitors have entered clinical trials, including BIBW2992, BMS-690514, and EKB-569. The efficacy has yet to be assessed (17). The combined application or single application of multiple-target drugs may block a variety of signaling pathways. The clinical trials in phase I/II (18) confirmed that the effective rate was up to $20 \%$ in the treatment of gefitinib resistance NSCLC using EGFR/VEGFR dual inhibitor erlotinib hydrochloride tablets combined with bevacizumab. Morgillo et al (19) found that T790M blocked gefitinib induced Bim upregulation and pro-apoptosis. L747 gene mutations were capable of weakening Bim upregulation and inhibit apoptosis of tumor cells (20). Therefore, to increase Bim expression or to activate its downstream targets may also be used in the treatment of NSCLC.

The study showed that the tumor response rate was $20.0 \%$ in the non-response group after the chemotherapy regimen was adjusted, suggesting that NSCLC was not only related to EGFR gene mutation but also related to other mechanisms such as VEGF gene mutation. The targeted therapy increased the response rate. The response rate of $\mathrm{CR}$ was $32.7 \%$ in the reaction group, the response rate of $\mathrm{PR}$ was $43.6 \%$, and the response rate of SD was $23.8 \%$. The drug resistance rates of CR, PR, and SD showed no statistical difference. However, the drug-resistant time of CR was significantly delayed and the difference was statistically significant, suggesting that the drug resistance was not related to the response degree, but the response time of better response degree was delayed. As discussed above, the mechanism of drug resistance on both the secondary mutation of EGFR gene and Met gene amplification may be related to the natural biological characteristics of the tumor. Gefitinib could interfere or delay but not completely block the tumor progression. Cancers may adapt to the drug treatment in diversed forms. However, blocking its intervention targets potentially leads to drug resistance or failure. After the chemotherapy regimen was adjusted, the response rates of $\mathrm{CR}, \mathrm{PR}$, and SD patients regained showed no statistical difference, $\sim 15 \%$, which was consistent with the reports of relevant literature. In conclusion, gefitinib-targeted therapy for NSCLC showed certain drug resistance, which may not be related to the response.

\section{References}

1. Cragg MS, Kuroda J, Puthalakath H, Huang DC and Strasser A: Gefitinib-induced killing of NSCLC cell lines expressing mutant EGFR requires BIM and can be enhanced by $\mathrm{BH} 3$ mimetics. PLoS Med 4: 1681-1689, discussion 1690, 2007.

2. Wu YL, Zhong WZ, Li LY, Zhang XT, Zhang L, Zhou CC, Liu W, Jiang B, Mu XL, Lin JY, et al: Epidermal growth factor receptor mutations and their correlation with gefitinib therapy in patients with non-small cell lung cancer: a meta-analysis based on updated individual patient data from six medical centers in mainland China. J Thorac Oncol 2: 430-439, 2007.

3. Xu Y, Liu H, Chen J and Zhou Q: Acquired resistance of lung adenocarcinoma to EGFR-tyrosine kinase inhibitors gefitinib and erlotinib. Cancer Biol Ther 9: 572-582, 2010.
4. Rosell R, Taron M, Sanchez JJ and Paz-Ares L: Setting the benchmark for tailoring treatment with EGFR tyrosine kinase inhibitors. Future Oncol 3: 277-283, 2007.

5. Costa DB, Halmos B, Kumar A, Schumer ST, Huberman MS, Boggon TJ, Tenen DG and Kobayashi S: BIM mediates EGFR tyrosine kinase inhibitor-induced apoptosis in lung cancers with oncogenic EGFR mutations. PLoS Med 4: 1669-1679, discussion 1680, 2007.

6. Uramoto H, Uchiumi T, Izumi H, Kohno K, Oyama T, Sugio K and Yasumoto K: A new mechanism for primary resistance to gefitinib in lung adenocarcinoma: the role of a novel G796A mutation in exon 20 of EGFR. Anticancer Res 27 (4B): 2297-2303, 2007.

7. Park J, McDonald JJ, Petter RC and Houk KN: Molecular dynamics analysis of binding of kinase inhibitors to WT EGFR and the T790M mutant. J Chem Theory Comput 12: 2066-2078, 2016.

8. Takeda M, Okamoto I and Nakagawa K: Survival outcome assessed according to tumor response and shrinkage pattern in patients with EGFR mutation-positive non-small-cell lung cancer treated with gefitinib or erlotinib. J Thorac Oncol 9: 200-204, 2014.

9. Inukai M, Toyooka S, Ito S, Asano H, Ichihara S, Soh J, Suehisa H, Ouchida M, Aoe K, Aoe M, et al: Presence of epidermal growth factor receptor gene $\mathrm{T} 790 \mathrm{M}$ mutation as a minor clone in non-small cell lung cancer. Cancer Res 66: 7854-7858, 2006.

10. Onitsuka T, Uramoto H, Nose N, Takenoyama M, Hanagiri T, Sugio K, Yasumoto K: Acquired resistance to gefitinib: the contribution of mechanisms other than the T790M, MET, and HGF status. Lung Cancer 68: 198-203 2010.

11. Engelman JA, Zejnullahu K, Mitsudomi T, Song Y, Hyland C, Park JO, Lindeman N, Gale CM, Zhao X, Christensen J, et al: MET amplification leads to gefitinib resistance in lung cancer by activating ERBB3 signaling. Science 316: 1039-1043, 2007.

12. Bean J, Brennan C, Shih JY, Riely G, Viale A, Wang L, Chitale D, Motoi N, Szoke J, Broderick S, et al: MET amplification occurs with or without T790M mutations in EGFR mutant lung tumors with acquired resistance to gefitinib or erlotinib. Proc Natl Acad Sci USA 104: 20932-20937, 2007.

13. Yano S, Wang W, Li Q, Matsumoto K, Sakurama H, Nakamura T, Ogino H, Kakiuchi S, Hanibuchi M, Nishioka Y, et al: Hepatocyte grow th factor induces gefitinib resistance of lung adenocarcinoma with epidermal growth factor receptor-activating mutations. Cancer Res 68: 9479-9487, 2008.

14. Velling T, Stefansson A and Johansson S: EGFR and beta1 integrins utilize different signaling pathways to activate Akt. Exp Cell Res 314: 309-316, 2008.

15. Ju LX, Zhou CC, Tang L, Zhao YM, Yang XJ, Su B, Meng SY, Li W, Yan LH and Ding YM: Drug resistance mechanism of non small cell lung cancer PC9/AB2 cell line with acquired drug resistance to gefitinib. Zhonghua Jie $\mathrm{He} \mathrm{He} \mathrm{Hu} \mathrm{Xi}$ Za Zhi 33: 354-358, 2010 (In Chinese).

16. Al-Temaimi R, Kapila K, Al-Mulla FR, Francis IM, Al-Waheeb S and Al-Ayadhy B: Epidermal growth factor receptor mutations in nonsmall cell lung carcinoma patients in Kuwait. J Cytol 33: 1-6, 2016.

17. Kwak EL, Sordella R, Bell DW, Godin-Heymann N, Okimoto RA, Brannigan BW, Harris PL, Driscoll DR, Fidias P, Lynch TJ, et al: Irreversible inhibitors of the EGF receptor may circumvent acquired resistance to gefitinib. Proc Natl Acad Sci USA 102: 7665-7670, 2005.

18. Herbst RS, Johnson DH, Mininberg E, Carbone DP, Henderson T, Kim ES, Blumenschein G Jr, Lee JJ, Liu DD, Truong MT, et al: Phase I/II trial evaluating the anti-vascular endothelial growth factor monoclonal antibody bevacizumab in combination with the HER-1/epidermal growth factor receptor tyrosine kinase inhibitor erlotinib for patients with recurrent non-small-cell lung cancer. J Clin Oncol 23: 2544-2555, 2005.

19. Morgillo F, D'Aiuto E, Troiani T, Martinelli E, Cascone T, De Palma R, Orditura M, De Vita F and Ciardiello F: Antitumor activity of bortezomib in human cancer cells with acquired resistance to anti-epidermal growth factor receptor tyrosine kinase inhibitors. Lung Cancer 71: 283-290, 2011.

20. Park IH, Kim JY, Jung JI and Han JY: Lovastatin overcomes gefitinib resistance in human non-small cell lung cancer cells with K-Ras mutations. Invest New Drugs 28: 791-799, 2010. 